\title{
Von Tauschringen und Kundenkarten
}

\author{
In den letzten Jahren erfreut sich bargeldloser Konsum im Rahmen von Tausch- \\ ringen wachsender Beliebtheit. Doch wie attraktiv ist solch ein Konzept \\ jenseits sozial-ökologischer Nischen? Und welche anderen Möglichkeiten gibt \\ es, die Rahmenbedingungen für nachhaltiges Konsumhandeln zu verbessern? \\ Ein Ansatzpunkt für entsprechende Innovationen, der zurzeit in einem Pra- \\ xisprojekt getestet wird, ist die Entwicklung einer nachhaltigen Kundenkarte.
}

$\mathrm{D}$ Bedeutung des Konsums für die Umsetzung des Leitbilds einer nachhaltigen Entwicklung ist mittlerweile unumstritten. Doch wie kann ein nachhaltiges Konsumverhalten konkret aussehen? Welche Angebote und Strukturen sind erforderlich, um entsprechende Verhaltensanreize zu setzen? Häufig wird in diesem Zusammenhang das Bild einer Alternativökonomie gezeichnet, in der Produkte und Dienstleistungen bargeldlos von privat zu privat getauscht werden. Doch wie attraktiv ist solch ein Konzept jenseits sozial-ökologischer Nischen? Und welche anderen Möglichkeiten gibt es, die Rahmenbedingungen für nachhaltiges Konsumhandeln zu verbessern? Im Rahmen der Entwicklung eines Zentrums für nachhaltige Dienstleistungen untersucht das Institut für ökologische Wirtschaftsforschung (IÖW) diese Fragestellungen und erarbeitet innovative Ansätze für die Vermarktung nachhaltiger Konsumangebote (1).

Ausgangspunkt ist eine Initiative verschiedener gemeinnütziger Organisationen aus der Region Heidelberg zum Aufbau eines Zentrums für nachhaltige Dienstleistungen im Stadtteil Bergheim. Mit der Etablierung eines derartigen Zentrums wird das Ziel verfolgt, durch das Angebot ökologisch effizienter Dienstleistungen, wie zum Beispiel Vermietung und gemeinsame Nutzung von Produkten, Reparatur oder Dienstleistungstausch, Umweltentlastungen und Ressourceneinsparungen zu erreichen. Zugleich soll die Stärkung von Eigeninitiative und bürgerschaftlichem Engagement, etwa durch nachbarschaftliche Unterstïtzung oder eine Freiwilligen-Börse einen Beitrag zur Verbesserung des sozialen Klimas und Erhöhung der Lebensqualität leisten.

Der Gesamtverbund besteht aus drei Bausteinen, die von unterschiedlichen Akteuren getragen werden (siehe Abbildung 1): der gemeinnützige Verein Weitergeben, unter dessen Dach die Praxispartner organisiert sind, die das Angebot des Zentrums stellen, und der das Weitergeben-Büro betreibt,

- das Agenda-Büro Heidelberg, verantwortlich fuir die Koordination des Praxisprozesses, und

- das IöW, verantwortlich für die Gesamtleitung und wissenschaftliche Begleitforschung sowie das prozessbegleitende Monitoring.

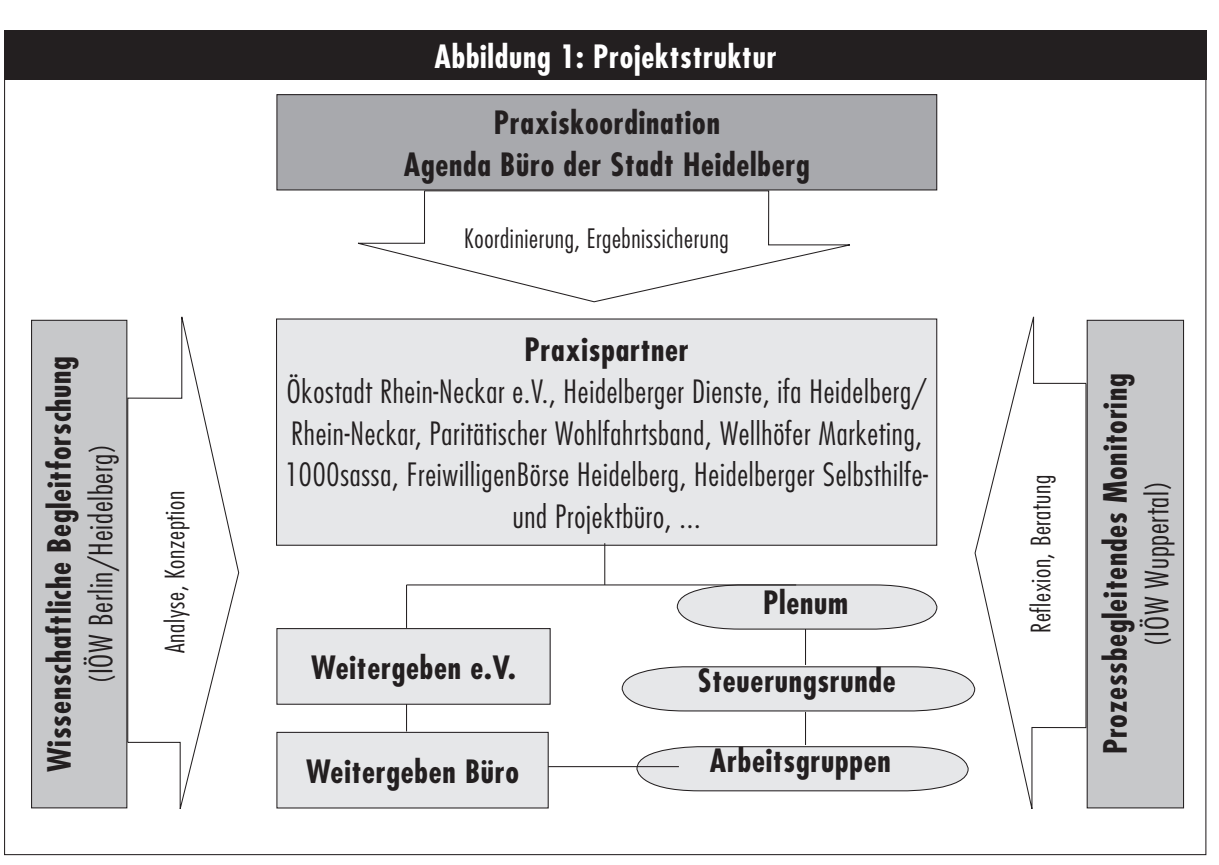

\section{Der Forschungsansatz}

Die Problemstellung des Vorhabens ist transdisziplinärer Natur. Sie kombiniert die Entwicklung innovativer Lösungskonzepte an der Schnittstelle von gesellschaftlicher Lebenswelt und Umwelt mit einem disziplinübergreifenden Forschungsansatz. Ersteres bezieht sich auf die Schaffung eines Netzwerks unterschiedlicher Praxisakteure mit dem Ziel öko-effiziente Nutzungsstrategien bereitzustellen sowie bürgerschaftliches Engagement und wird die Komplexität des Projekts abermals unterstrichen. „Offenheit“ soll in diesem Zusammenhang darauf hinweisen, dass es darum geht, in einen - auch im Sinne der Einbindung weiterer Akteure - offenen Dialog und Konsultationsdiskurs einzutreten. In dessen Verlauf sollen gemeinsam das im Rahmen des Zentrums bereit zu stellende Leistungsbündel und seine Präsentationsformen abgestimmt, entwickelt, umgesetzt und dynamisch erweitert werden. nachbarschaftliche Hilfe zu fördern. Der Forschungsansatz setzt einen Methodenmix aus empirischer Sozialforschung (Repräsentativbefragung, Gruppendiskussionen), Umweltbewertungsverfahren (orientierende Ökobilanzen) sowie regional- und betriebswirtschaftlichen Analysen ein.

Der gewählte Ansatz ist insofern als innovativ zu betrachten, als klassische Methoden der Marktforschung (Umfragen, Fokusgruppen, zielgruppenorientierte Leistungsentwicklung) im Kontext sozial-ökologischer Konsumforschung angewendet werden. Wenngleich die beteiligten Praxisakteure, allesamt aus dem Non-Profit-Bereich, in ihren jeweiligen Arbeitsfeldern ausgesprochen professionell agieren, stellt die kooperative Auseinandersetzung mit dem gewählten Ansatz eine Herausforderung dar.

Durch die Tatsache, dass das Vorhaben als offener, im Zeitablauf sich ausdifferenzierender Prozess gestaltet ist, an dem je nach Arbeitsphase und Schwerpunktsetzung unterschiedliche Akteure in unterschiedlicher Intensität partizipieren, 


\section{- Die Initiierungsphase}

Im Frühjahr 2002 war das Zentrum im Wesentlichen als Vermittlungsdienst für den bargeldlosen, privaten Tausch von Produkten und Dienstleistungen, ähnlich einem Tauschring, konzipiert. Parallel zur Entwicklung dieses Konzepts wurde im Rahmen der wissenschaftlichen Begleitforschung eine repräsentative Telefonumfrage unter 500 Heidelberger Bürgern durchgeführt, auf deren Basis mittels einer Clusteranalyse potenzielle Zielgruppen ermittelt wurden. Vertreterinnen und Vertreter der einzelnen Gruppen wurden für Kreativ-Workshops rekrutiert. Auf diesen jeweils vierstündigen Veranstaltungen wurden die Idee des Zentrums, sein damals aktuelles Leistungsspektrum sowie der öffentliche Auftritt, der bis dahin einen Flyer, die Weitergeben-Zeitung, einen Internetauftritt sowie eine Geschäftsstelle umfasste, kritisch reflektiert und Verbesserungsmöglichkeiten erörtert.

Zwar konnten mit dem damaligen Konzept finanzielle Vorteile durch das Güterteilen sowie eine Intensivierung nachbarschaftlicher Kontakte assoziiert werden. Insgesamt überwogen aber quer über alle Zielgruppen - die wahrgenommenen Nachteile. Dazu zählten unter anderem ein allgemeines Misstrauen gegenüber einer institutionalisierten Kontaktagentur, die Gefahr der Bürokratisierung von bislang informellen Unterstuitzungsleistungen sowie der fehlende Vertrauensbezug in Bezug auf den Güter- und Dienstleistungsaustausch mit Fremden. Desweiteren wurde Regelungsbedarf für Haftungs- und Versicherungsfragen gesehen und mehr Flexibilität bei der zu erbringenden Gegenleistung gefordert, entweder durch Eigenleistung, Leihgeräte oder ein finanzielles Entgelt.

\section{Einen Überblick über alle bisher erschienenen Hefte des Informationsdienstes}

\section{"Ökologisches Wirtschaften"}

finden Sie auf unseren Internetseiten
unter der Adresse: www.oekom.de

Wir freven uns auf Ihren Besuch!

\section{Der Relaunch}

Das kritische Feedback durch die potenziellen Nutzerinnen und Nutzer des Zentrums war Gegenstand eines Workshops „Ideenentwicklung“ mit den Praxisakteuren im Sommer 2002, auf dem mit Hilfe von Kreativ-Methoden wie der Collagentechnik eine Neupositionierung des Zentrums erarbeitet wurde. Dabei war es Konsens,

- den Servicecharakter des Zentrums zu Lasten seiner Rolle als „Kontaktagentur“ zu stärken,

- das Angebotsspektrum durch innovative Leistungen zu erweitern, beispielsweise einen Reparaturservice,

- die Wirtschaftlichkeit unter anderem durch kommerzielle Angebote zu verbessern sowie

- den Zugang für unterschiedliche Kundensegmente zu erleichtern.

Zentrale Innovationsidee, neben einem Vermittlungsservice für die Reparatur von Elektrogeräten, war eine Kundenkarte für nachhaltige Produkte und Dienstleistungen.

\section{Kundenkarte als zentrales Instrument}

Die Kundenkarte ist mittlerweile Gegenstand intensiver Entwicklungsaktivitäten, die im Frühjahr 2003 zum Relaunch des Zentrums führen sollen. Grundidee der Kundenkarte ist die Bildung einer Werbegemeinschaft für nachhaltige Produkte und Dienstleistungen aus der Region Heidelberg mit dem Ziel, die Kundenbindung zu erhöhen und damit den Absatz zu steigern. Kartennutzer sollen eine jährliche Gebühr an den Kartenbetreiber zahlen und erhalten dafür bei den angeschlossenen Leistungspartnern Rabatte. Geplante Leistungspartner sind unter anderem Naturkostläden und Gerätevermieter.

In Fokusgruppen wurde dieses neue Konzept abermals zur Diskussion gestellt. Als positive Aspekte wurden hierbei die möglichen finanziellen Einsparungen, das breite Spektrum der Geschäfte, die ökologische Ausrichtung, die Unterstützung des Fachhandels und der kleinen Betriebe, der regionale Bezug zu Heidelberg sowie der Neuigkeitscharakter des Angebots wahrgenommen. Angesichts vieler kostenloser Karten wurde die geplante Jahresgebühr kritisch eingeschätzt und erschien die Rentabilität fraglich.

\section{- Fazit auf halbem Wege}

Nach einem guten Jahr Laufzeit hat das Forschungsvorhaben die Tragfähigkeit des Netzwerks mehrfach unter Beweis gestellt. Vor dem
Hintergrund der Ergebnisse der Marktforschung haben die Praxisakteure eine Neupositionierung der Initiative vorgenommen. Das bedeutet einerseits Kontinuität: Die Vereinsaktivitäten mit Stadtteilbezug sollen ausgebaut und intensiviert werden. Die Tauschaktivitäten stehen zwar nicht mehr im Mittelpunkt des Dienstleistungszentrums, werden jedoch für Interessierte mittels eines beteiligten Praxispartners nach wie vor angeboten, nämlich als Konsumgüter-Sharing von Ökostadt RheinNeckar. Das bedeutet andererseits aber auch Wandel: Die Entwicklung und Realisierung innovativer Angebote wie die nachhaltige Kundenkarte und den Reparaturvermittlungsservice, die konsequente Orientierung an breiteren Marktsegmenten sowie, damit eng verbunden, die räumliche Öffnung in Richtung ganz Heidelberg.

Die wissenschaftliche Begleitforschung nimmt hierbei eine Zwitterrolle ein: Einerseits wichtiger Impulsgeber für die Weiterentwicklung des Zentrums und damit ebenfalls eine Art Praxispartner. Andererseits „,neutrale“ Forschungsinstanz mit dem Untersuchungsgegenstand nachhaltige Dienstleistungen in regionalen Netzwerken. Der damit verbundene Spagat scheint charakteristisch für die sozial-ökologische Forschung. Welche Chancen und Risiken damit verbunden sind, soll gegen Ende des Vorhabens systematisch reflektiert werden.

\section{Anmerkung}

(1) Weitere Informationen auf www.ioew.de/dienstleistung unter dem Projekttitel "Nachhaltige Dienstleistungen in der Region". Das vom Bundesministerium für Bildung und Forschung (BMBF) im Rahmen des Förderschwerpunkts "Möglichkeiten und Grenzen neuer Nutzungsstrategien" geförderte Vorhaben startete im Dezember 2001 und endet im Mai 2004.

\section{Der Autor}

Gerd Scholl ist wissenschaftlicher Mitarbeiter im Forschungsfeld Ökologische Produktpolitik des Instituts für ökologische Wirtschaftsforschung (IÖW). Kontakt: IÖW, Potsdamer Str. 105, 10785 Berlin. Tel. 030-884594-20, Fax 030-8825439, E-Mail: gerd.scholl@ioew.de 
(c) 20I0 Authors; licensee IÖW and oekom verlag. This is an article distributed under the terms of the Creative Commons Attribution Non-Commercial No Derivates License (http://creativecommons.org/licenses/by-nc-nd/3.o/), which permits unrestricted use, distribution, and reproduction in any medium, provided the original work is properly cited. 